[Manuscript submitted for publication as an article in Mol. Cryst. \& Liq. Cryst., OLC-2009]

\title{
MORPHOLOGY, LINEAR AND NONLINEAR OPTICAL RESPONSE OF OCTOPOLAR CHROMOPHORES EMBEDDED IN A SILICA SONOGEL MATRIX
}

\author{
V. Torres-Zúñiga ${ }^{1 *}$, O.G. Morales-Saavedra ${ }^{1}$, G. Hennrich ${ }^{2}$, \\ J.O. Flores-Flores ${ }^{1}$ and R. Ortega-Martínez ${ }^{1}$. \\ 1) Centro de Ciencias Aplicadas y Desarrollo Tecnológico, Universidad Nacional Autónoma de \\ México, CCADET-UNAM. A. P. 70-186, Coyoacán, 04510, México, D. F. México. \\ 2) Departamento de Química Orgánica, Universidad Autónoma de Madrid, Cantoblanco, 28049 \\ Madrid, Spain. \\ * e-mail: vicentz@gmail.com
}

\begin{abstract}
In this work, a liquid crystalline octopolar molecule 1,3,5-tris(ethynylphenyl)benzene-(1) was successfully embedded as dopant chromophore within a $\mathrm{SiO}_{2}$ based sonogel network in order to prepare stable solid state hybrid materials with intense second harmonic generation (SHG). This recently synthesized octopolar compound exhibit a centrosymmetric $C_{3}$-symmetry structure, which is constituted by an electron-rich core and electron-poor periphery groups. The sonogel method, induced by energetic ultrasonic (US) waves applied at the TEOS $/ \mathrm{H}_{2} \mathrm{O}$ reactant mixture interface has proven to be a suitable route in the manufacture of monolithic hybrid systems suitable for optical characterizations. The octopolar-based hybrid samples were comprehensively studied in their morphology, spectroscopic, linear and second-order nonlinear optical (NLO) properties, according to several characterization techniques: epimicroscopy, AFM, UV-Vis- and fluorescence (PL) spectroscopies, and the NLO-SHG-technique. Epimicroscopy, photoluminiscent and absorption spectra evidence an optimal and homogeneous inclusion of the octopolar compounds within the glassy sonogel network. Without necessity of molecular poling processes, the effective quadratic-NLO susceptibility $\chi_{\text {effs }}^{(2)}$ of the hybrid was estimated to $13.6 \times 10^{-3} \mathrm{pm} / \mathrm{V}$ at $1064 \mathrm{~nm}$ by SHG-transmission experiments recorded far-off of the resonance regime.
\end{abstract}

Keywords: Liquid Crystals, Octopolar compounds, Sol-Gel, Hybrid Materials, Optical Properties. 


\section{Introduction}

Over the last two decades, substantive efforts have been dedicated to the synthesis of molecules with enhanced quadratic nonlinear optical effects, such as second harmonic generation (SHG). It is widely assumed that these molecular structures and the packing arrangement must be non-centrosymmetric, e.g. rodlike dipolar systems. Nevertheless, second-order ONL effects may also arise from some centrosymmetric molecular structures, such as octopolar compounds, which posses a zero overall dipole moment and liquid crystalline (LC) behavior in columnar phases [1-3]. The development of octopolar nonlinear optical materials suitable for several applications in optical, nonlinear optical and electro-optical devices is a topic of current interest among many research groups. For instance, the use of these structures plays an important role in the improvement of semiconductor elements in organic field-effect transistors or in organic light-emitting diodes, among others practical areas [4-5]. The octopolar structures present many other advantages in comparison to the more conventional dipolar molecules. For example, most commonly conjugated dipolar systems, in a supramolecular arrangement, such as a bulk format, can lead to extinction of the macroscopic susceptibility due to anti-parallel alignment of the molecules [2]. In contrast, octopolar molecules, possessing a zero overall dipole moment in both the ground and excited states [6], can ideally be arranged in the bulk phase in a way that their molecular susceptibility is additive [7]. Moreover, the octopoles have a broader range of tensorial coefficients than dipolar molecules, this lead to the observation of optimal second-order nonlinear optical responses which are polarization-independent with the light input of the laser source [8]. These and others features have directed increasing attention to the development of novel molecular octopolar chromophores.

On the other hand, the sol-gel technique has been widely and continuously used as a popular methodology to confine and encapsulate diverse organic and inorganic dopant species into highly inert, transparent and amorphous inorganic glassy networks. In fact, solid-state hybrid systems can be easily obtained by diverse sol-gel routes in order to prepare advanced materials suitable for optical characterizations and applications, with special interest in the linear and nonlinear optical processes [9-11]. Thus, in the case of organic-inorganic composites, the constituting organic compounds (usually provided in powder samples by the chemist colleges) will contribute with the desired optical property, whereas the inorganic part (glassy network) increases the mechanical stability and thermal strength of the system. Moreover, the optimal inclusion of organic chromophores into an amorphous sol-gel glass allows the fabrication of a wide variety of low-cost devices required for photonic purposes in several formats, as for example optical wave-guiding systems where the deposition of high 
quality organic thin films is essential to fill up the corresponding optical channels and circuitry of these devices, which have to remain stable for long periods of time [12]. Yet, only correct combinations of sol-gel/dopants can produce homogeneous and stable chromophore dispersion into the glassy network, however still appropriate doping control can be achieved in order to produce several hybrids with different doping rates and chromophore loading.

In this work, we report on the development of octopole $/ \mathrm{SiO}_{2}$-sonogel based solid state hybrid materials implementing a recently developed octopolar compound synthesized in our research group via Sonogashira coupling: 1,3,5-tris(ethynylphenyl)benzene-(1) [13]. The morphological, linear and nonlinear optical characterization of the octopole/sonogel hybrids were performed in order to explore the spectroscopic and optical performance of this compound within the sonogel environment, exploiting the catalyst-free (CF) sonolysis route to produce highly pure (chemically and optically) solgel samples generated by ultrasonic irradiation (sonogel materials). The experimental results shown in this paper have been carried out as a primary and explorative work in the development of photonic organic-inorganic prototypes, where the inclusion of multipolar molecules into the sonogel networks plays an important role. This may provide practical alternatives for the development of novel and low cost optical materials.

\section{Experimental section}

\subsection{Chemical structure of the implemented NLO-LC octopole}

So far, the principal charge template to design $\chi^{(2)}$ NLO-active octopolar molecules in 2D structures is to synthesize noncentrosymmetrically substituted trigonal $\pi$-conjugated systems that display efficient charge transfer from the periphery to the center of the molecule, where the core of the octopolar system may be a benzene-like molecule [13] or even a single atom (e.g. crystal violet [14]). Thus, the implemented 1,3,5-tris(ethynylphenyl)benzene-(1) systems constitute among octopolar NLO chromophores a family with remarkable second-order polarizabilities and liquid-crystalline discotic mesophases [13]. This compound exhibits an electron-rich benzene core and an electron-poor periphery, which represents a non-common octopolar structure, see Fig. 1. Indeed, this inversion of the functional groups seems to be more appropriate for efficient intra-molecular charge transfer [13]. According to semi-empirical calculations (performed in ACD/ChemSketch ${ }^{\mathrm{TM}}$ software, V. 12.0), this molecule present a refractive index of 1.70 and a molecular polarizability of $63.38 \pm 0.510^{-24} \mathrm{~m}^{3}$. 


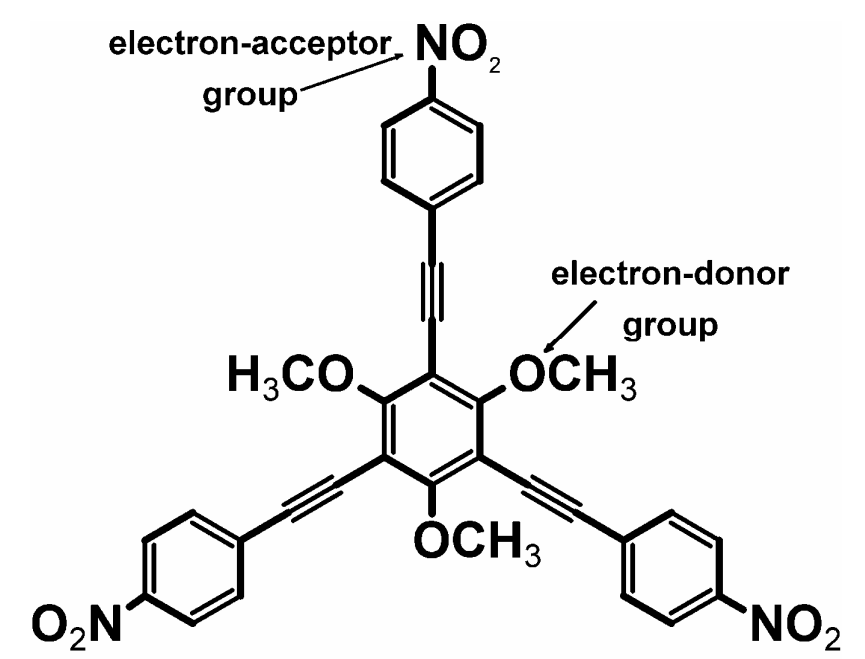

Figure 1. Chemical structure of the implemented 1,3,5-tris(ethynylphenyl)benzene-(1) octopole. The center of the molecule is composed by donor-groups, whereas the molecular periphery is composted by acceptor-groups.

\subsection{Synthesis of a catalyst-free sonogel network as a host material for LC octopoles}

The sol-gel method is frequently used to synthesize amorphous $\mathrm{SiO}_{2}$ glasses, this route is based on the hydrolysis of different precursors such as tetraethyl-orthosilicate (TEOS) and tetramethoxysilane (TMOS), followed by condensation reactions of the hydrolyzed species. This procedure has been widely adopted as an appropriate process to obtain glassy doped materials at room temperature with stable amorphous structure and high transparency. Both, hydrolysis and condensation reactions normally occur in the presence of acidic or basic catalysts, where ethanol and methanol are common solvents for the precursors and water reactants. Theoretical and experimental details for the sol-gel synthesis of $\mathrm{SiO}_{2}$ can be found extensively in the literature $[15,16]$. However, in this contribution, the use of both solvents and catalysts is evaded at all. The hydrolyzed species are substituted by molecular radicals generated by energetic ultrasound pulses, the obtained sonolyzed-gel materials are free of solvents or impurities remanents, and present an elastic modulus several orders of magnitude higher that those prepared by conventional methods [17]. In the present article, only a brief explanation of this methodology is presented in order to clarify the procedure to obtain $\mathrm{CF}-\mathrm{SiO}_{2}$ sonogel glasses as host materials for organic compounds and the development of CF-hybrid composites. Extensive details on the synthesis, chemistry and methodology of this sonogel can be found in recent literature [18]. 
The synthesis of the CF-sonogel consists basically on the following steps: 1) As precursor reactants $25 \mathrm{~mL}$ of TEOS (Fluka 99\% purity) and $25 \mathrm{~mL}$ of distilled water are mixed into a glass vessel and stabilized at $1^{\circ} \mathrm{C}$ for $1 \mathrm{~h}$ before ultrasonic (US)-irradiation (these reactants are non-soluble). 2) A metallic ultrasound tip (Cole-Parmer-CPX, with $1.25 \mathrm{~cm}$ in diameter), carefully located at the TEOS $/ \mathrm{H}_{2} \mathrm{O}$ surface interface provides an effective irradiation power density on the order of $\sim 3.2 \mathrm{~W} \mathrm{~cm}^{-3}$ working at $20 \mathrm{KHz}$. The tip of the ultrasonic-wave generator also acts as an ultrasonichomogenizer. After $3 \mathrm{~h}$ of programmed US-irradiation (on/off -intermittent sequences of $5 \mathrm{~s}$; net irradiation time: $1.5 \mathrm{~h}$ ), the sonicated suspension was kept in the reactor vessel at room conditions for $24 \mathrm{~h}$; thereafter two immiscible phases come out: the upper one, corresponding to unreacted TEOS, which is removed and eliminated; whereas the more dense lower phase (sol-phase) corresponding to a stable colloidal suspension and containing the sonicated induced hydrolyzed product (OH-TEOS), is capable to produce (after drying and condensation) a highly pure $\mathrm{SiO}_{2}$ amorphous network.

In fact, in order to fabricate organic-inorganic hybrid glasses, the chromophore doping must be performed in the liquid sol-phase; hence, the dopant solution should be previously prepared. It has been noted that tetrahydrofuran (THF) based organic dopant solutions perform well in order to develop the hybrid structures $[11,18]$. This chromophore solution (in our case: $0.36 \mathrm{mg}$ of octopole powder dissolved in $3 \mathrm{~mL}$ of THF) can be afterwards added and ultrasonically mixed to the OH-TEOS colloidal suspension in order to start the inclusion of dopants within the porosity of the forming $\mathrm{SiO}_{2}$ glass.

Prepared hybrid materials were generated with a starting total volume of $2 \mathrm{~mL}$, the $\mathrm{OH}-$ TEOS:Dopant concentration ratio was set to 1.5:0.5 mL. Undoped sonogel samples (2.0:0.0) were also prepared for reference and calibration purposes. The samples were isolated with a plastic cover to avoid atmosphere and temperature variations and conserved for about two weeks at room conditions in closed recipients with a small hole on the cap in a clean-dry-dark environment. Afterwards, the drying process of the samples was completed and the formation of bulk samples is achieved. Monolithic cylindrical samples with good optical quality, diameters of $\sim 7 \mathrm{~mm}$ and thickness of $\sim 1.2 \mathrm{~mm}$ were obtained after this procedure. These hybrid and reference samples were investigated in their optical, non-linear optical, morphological and spectroscopic properties. 


\subsection{Physical characterization techniques}

Standard characterization techniques were applied to undoped reference and hybrid sonogel samples in order to determine their structural and optical properties:

i) Morphological AFM and Epi-microscopy study: The surface morphology of the composites was studied in intermittent contact mode by AFM (autoProbe CP, Scannig Probe Microscope, Park Scientific Instruments) in order to evaluate the homogeneity and possible superficial flaws at different scales. The sample quality was also monitored via reflection microscopy (epi-microcopy), with a digital microscope (HW10x450, TTS-Unlimited) and a CCD-camera.

ii) Spectral UV-Vis analysis: Linear optical absorption spectra in reference and hybrid samples were systematically obtained in the $200-1100 \mathrm{~nm}$ range using a double beam Shimadzu UV-vis spectrophotometer, using air in the reference beam.

iii) Photo-luminescent analysis: Photo-luminescent (PL) spectra were obtained with a FluoroMax-3, Jobin_Yvon-Horiba fluorimeter and the excitation wavelength was set to $\lambda_{\text {exc }}=373 \mathrm{~nm}$, according to the absorption spectral analysis of the sample. Both UV-Vis and PL studies brings essential information concerning the electronic properties of the guest molecule within the sonogel environment.

iv) Quadratic non-linear optical analysis: The second-order optical nonlinearity of the hybrid films were determined by the SHG-technique on transmission measurements. The SHG experimental device is schematically shown in Fig. 2. The set-up is constituted by a commercial Q-switched $\mathrm{Nd}$ :YAG Laser system (Surelite II from Continuum, $\lambda_{\omega}=1064 \mathrm{~nm}$, with repetition rate of $10 \mathrm{~Hz}$ and a pulse width of $\tau \sim 22 \mathrm{~ns}$ ), which provides the fundamental wave. Typical pulse powers of $\sim 100 \mu \mathrm{J}$ were filtered in order to irradiate the samples by means of a $f=5 \mathrm{~mm}$ focusing lens, thus, peak irradiances in the order of $15 \mathrm{MW} / \mathrm{cm}^{2}$ were achieved at the focal spot on the hybrid composites. This value was slightly below the energy damage threshold supported by the samples under strong focused beam irradiation. The polarization of the fundamental beam (parallel $\boldsymbol{P}$ or perpendicular $\boldsymbol{S}$ polarizing geometry) was selected by means of an IR-coated Glan-Laser polarizer and after a $\lambda / 2$-Quarterretarder. A second polarizer was used as analyzer allowing the characterization of the SHG signals. The second harmonic waves (at $\lambda_{2 \omega}=532 \mathrm{~nm}$ ) were detected by a sensitive photomultiplier tube (Hamamatsu R-928) placed behind interferential optical filters (centered at $532 \pm 5 \mathrm{~nm}$ ). The SHG device was calibrated by means of a $Y$-cut $\alpha$-quartz crystal, wedged in the $\mathrm{d}_{11}$-direction $\left(\mathrm{d}_{11}=0.64\right.$ $\mathrm{pm} / \mathrm{V}$ ), which is commonly used as second order NLO reference $[19,20]$. When absorption effects are neglected, the ratio between effective $\chi_{\text {eff }}^{(2)}$ SHG over the $\chi_{q}^{(2)}$ of the reference quartz is [21]: 


$$
\frac{\left|\chi_{e f f_{s}}^{(2)}\right|}{\left|\chi_{q}^{(2)}\right|} \approx \frac{L_{q c}}{L_{s}} \sqrt{\frac{I_{s}}{I_{q}}},
$$

here $L_{q c}$ is the coherence length of the reference crystal $(\sim 20.6 \mu \mathrm{m}), L_{S}$ is the sample thickness $(\sim 1.2 \mathrm{~mm}), I_{s}$ and $I_{q}$ are the maximum SHG-signal intensities of the sample under study and the reference quartz crystal, respectively. Thus, this elementary relation can be used in order to roughly estimate the quadratic susceptibility of a new material relative to a reference one.

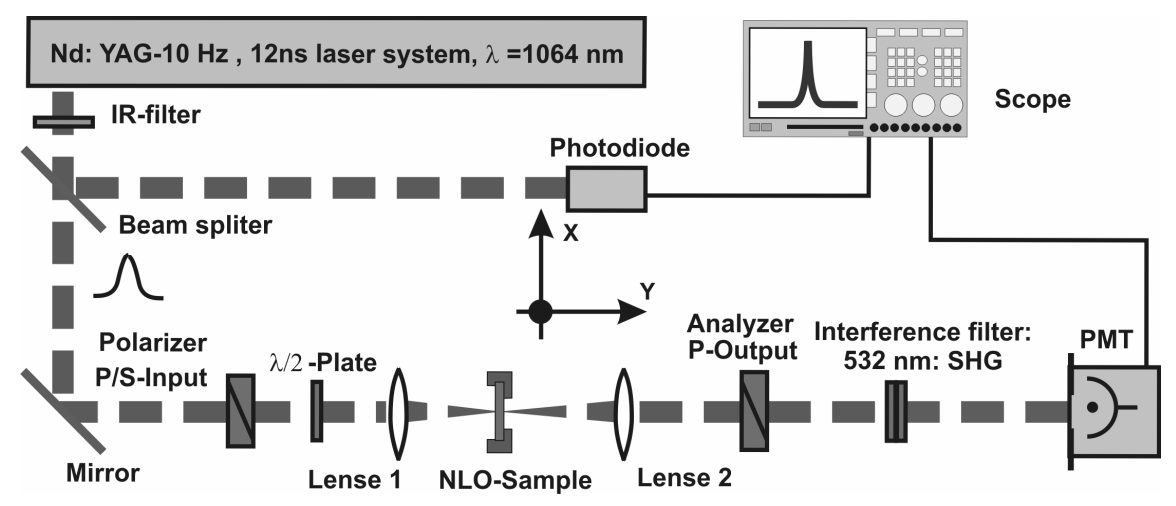

Figure 2. Experimental set-up implemented to measure the SHG-transmission in solid state sonogel hybrid samples.

\section{Results and discussions}

\subsection{Morphological AFM-analysis}

The hybrid bulk samples present a rigid and uniform cylindrical structure. According to the epi-microscopy images (see Fig. 3), the developed octopolar based hybrids present in an intense yellowish color confirming the inclusion of the chromophore within the sonogel network. The sample shows relatively small inhomogeneities (with largest diameters of $\sim 50 \mu \mathrm{m}$ ) that may be produced by the irregular evaporation and shirking process occurring in different regions of the sample; nevertheless, still good optical quality and uniformity can be achieved in order to perform optimal optical characterizations. In fact, laser beams used in different photophysical characterization experiments typically exhibit beam spots of $\sim 1 \mathrm{~mm}$; hence, these tiny inhomogeneities can be considered as negligible in the sense that the obtained optical signals rather represent an averaged output response of the light-matter interaction. In our case however, the spot size dimension of the focused laser beam implemented for NLO-SHG-experiments, precisely match the same length scale of the largest material inhomogeneities, making the SHG-response more sensitive to local defects or 
inhomogeneities. In any case, for most of the practical applications, the quadratic NLO-signals can be interpreted in terms of an average molecular response obtained from the bulk material to the laser excitation. Thus, we can argue that the spot dimension of our laser beam implemented for SHGexperiments is in the lower limit to generate reproducible experimental data, as will be shown in next sections.

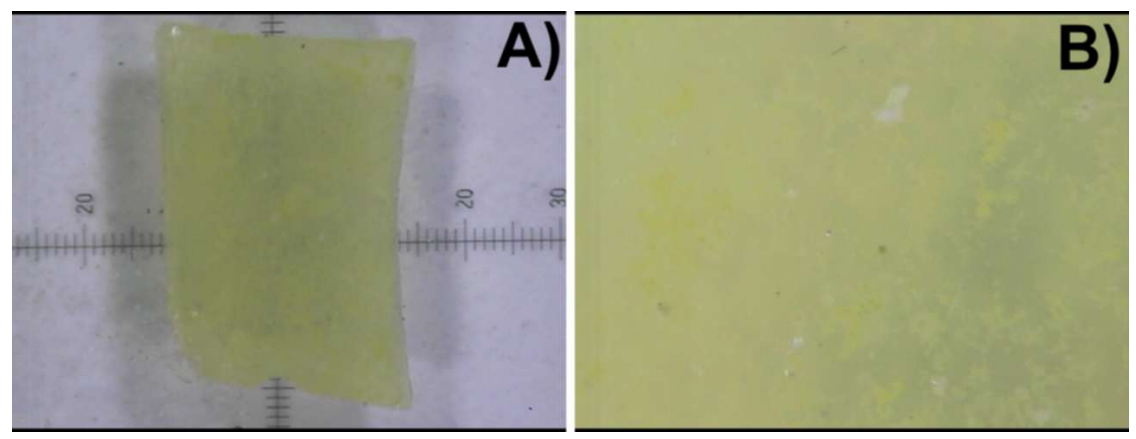

Figure 3. Epimicroscopy-picture of a octopole/sonogel hybrid composite at magnification optical powers of: A) 15X, and B) 350X. Note the typical yellow color of the doped glass, characteristic of the octopolar compound, indicating optimal chromophore doping.

On the other hand, AFM micrographs confirm again the inclusion of the octopolar chromophores within the $\mathrm{SiO}_{2}$ matrix (see Fig. 4). Indeed, AFM-images show an increment of the average surface roughness of about $200 \%$ from the pure reference sample to the hybrid composite, this remarkably roughness increment is due to the chromophore loading of the sonogel nano-pores, which causes irregularities in the surface grains.
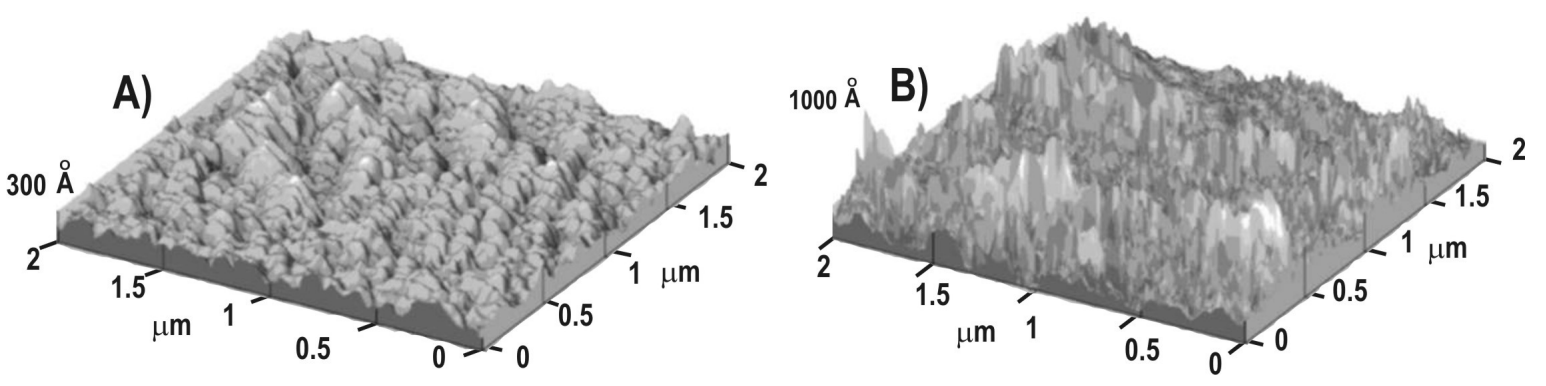

Figure 4. 3D-AFM-micrographs showing the surface morphology of sonogel samples: A) a pure-undoped reference sonogel sample (2.0:0.0), and B) an octopole based hybrid glass (1.5:0.5). 


\subsection{UV-Vis and photoluminescent spectroscopic measurements}

Figure 5 shows the comparative absorption spectra of a moderated (1.5:0.5)-doped composite, a (2.0:0.0)-reference sample, a THF/octopole solution, and the THF-solvent. The spectrum of the THF-solvent presents a sharp and intense absorption band in $277 \mathrm{~nm}$ and is fully transparent at optical wavelengths. Similarly, the pure reference sonogel shows a low absorption at optical wavelengths and an intense band to the UV, typical of $\mathrm{SiO}_{2}$ glassy materials. Thus, it is guaranteed that the addition of optical colored dopant chromophores dissolved in THF and embedded within the sonogel environment would be easily detected in the UV-spectra. In fact, a distinctive band of the implemented chromophore can be recognized in both the octopolar based THF-solution (band at $371 \mathrm{~nm}$ ) [13], and in the solid-state composite (wider band centered at $386 \mathrm{~nm}$ ). This band shows a monotonically decreasing tail indicating certain conjugation degree of the material. Specifically, in octopolar systems, these absorption bands above $350 \mathrm{~nm}$, are indicative of efficient charge transfer from the electron-rich benzene core to the electron-deficient periphery [14]. Here, a bathochromic shift is observed form the liquid to the solid-state phase which is probably caused by the formation of Jaggregates, which is a kind of $\pi-\pi$ electron stacking of the chromophores in the matrix [22].

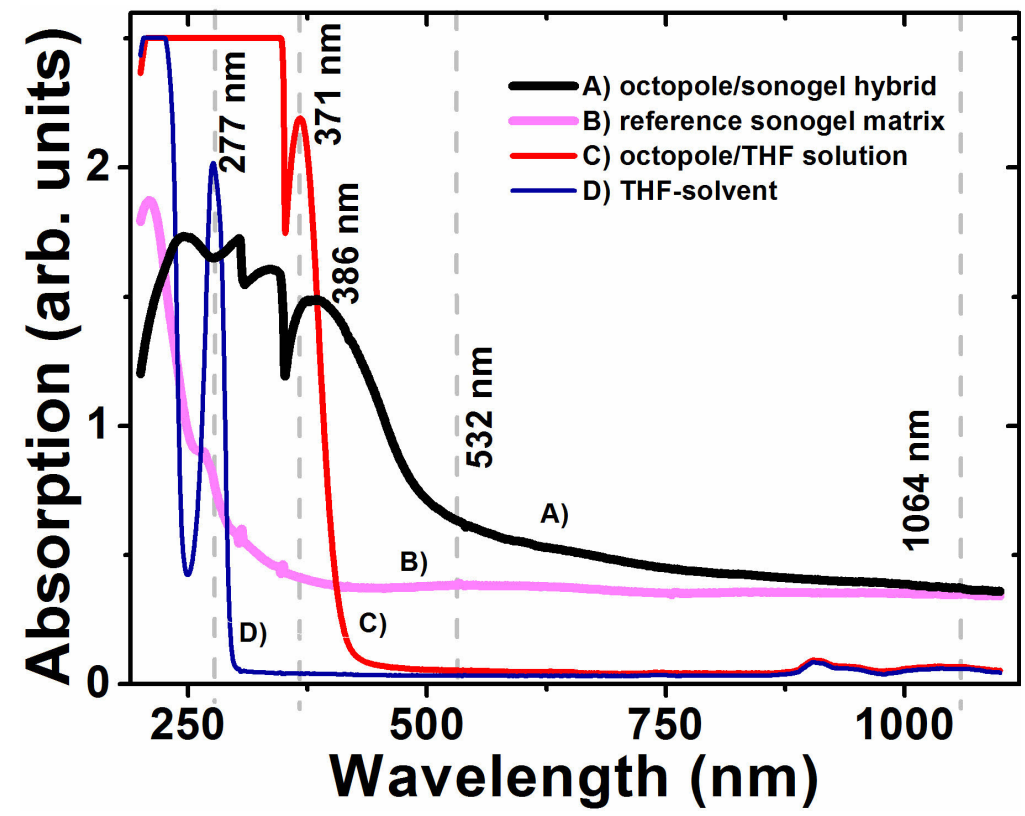

Figure 5. Comparative UV-Vis absorption spectra of a reference and an hybrid material: A) octopole/sonogel hybrid (1.5:0.5), B) reference sonogel matrix $(2.0: 0.0), \quad$ C) octopole/THF solution, and D) pure THF solution.

The evident saturation of the absorption band with increasing doping levels of the samples, demonstrates a favorable inclusion of the octopolar compounds within the $\mathrm{SiO}_{2}$ network (not showed 
her for simplicity of the graph). Is worthy to note that absorption of the hybrid composite at both fundamental and doubled laser frequencies $\left(\omega=1064 \mathrm{~nm}\right.$, and $\omega_{2}=532 \mathrm{~nm}$, respectively) are not in the resonance regime; this means that the absorption effects in SHG can be neglected and that Eq. (1) can reasonably be applied for the relative evaluation of the $\chi_{\text {effs }}^{(2)}$ coefficient of hybrid materials.

Figure 6 shows a comparative photoluminiscent spectra of the studied materials recorded from 200 to $900 \mathrm{~nm}$ (excitation wavelength was set at $\lambda_{\text {exc }}=373 \mathrm{~nm}$ according to the resonance absorption of the hybrid samples). It is shown from this figure that the PL-spectrum of a pure reference sample exhibit a weak and broad emission around 350-750 nm, which is typical of $\mathrm{SiO}_{2}$ based glasses $[15,16]$. On the other hand, in the octopole/THF solution present a weak and broaden peak around $510 \mathrm{~nm}$. Finally, the octopole/sonogel hybrid exhibits a well defined and structured photoemission in the visible optical range, where typical features of the glass network can strongly be detected; in fact the main PL-activity which distinguishes the emission of the hybrid system from the reference glass is a broader and higher PL-activity within the $500-700 \mathrm{~nm}$ spectral range.

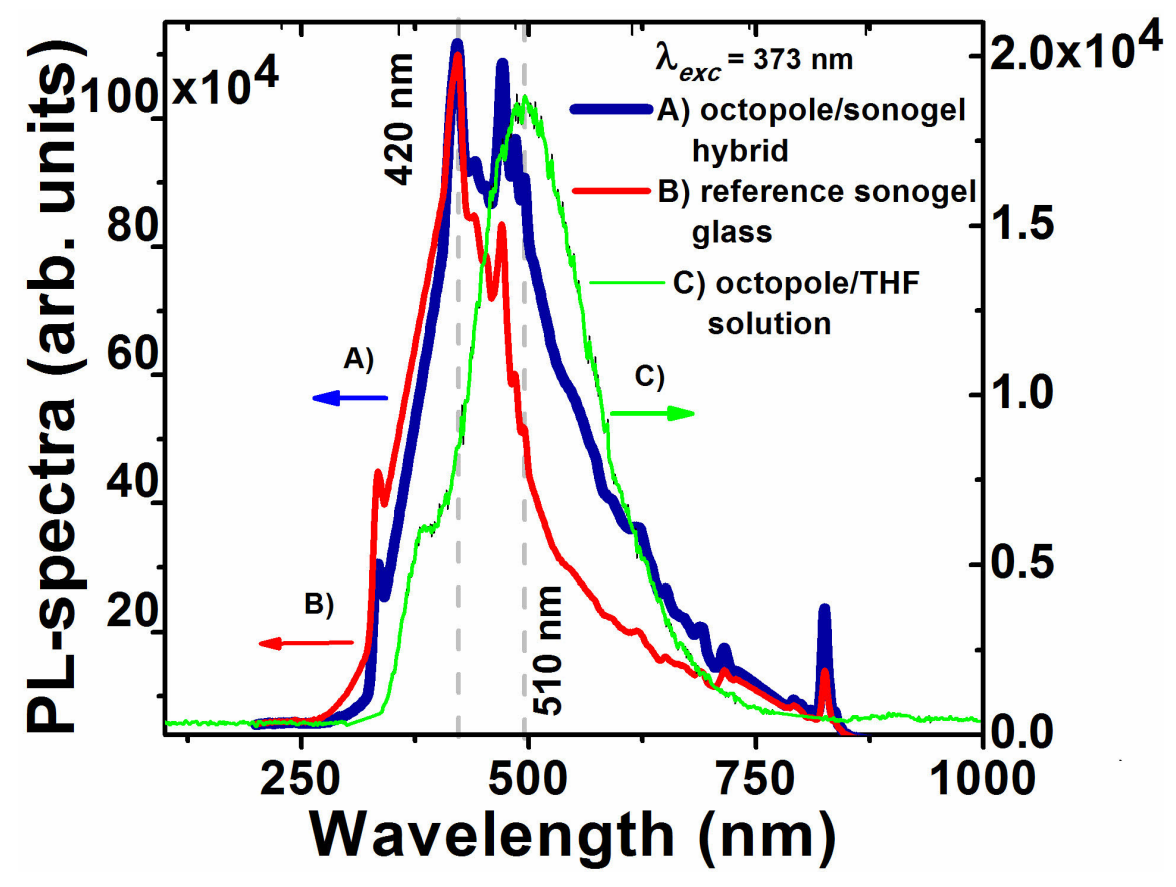

Figure 6. Comparative PL-spectra obtained from the solid state phase: A) reference sonogel glass, B) moderate doped octopole/sonogel sample, and C) the liquid octopole/THF phase. Excitation wavelength was set to $\lambda_{\text {exc }}=373 \mathrm{~nm}$. 


\subsection{NLO-SHG measurements}

Figure 7 illustrates a SHG activity of the octopole/sonogel hybrid system; it shows typical SHG-measurements directly taken from the oscilloscope screen (at normal laser incidence) and performed in P-In/P-Out laser beam polarization geometry. Since the amorphous sonogel does not contribute in the quadratic-NLO emission due to its inherent amorphous centrosymmetric glassy structure, it is assured that the SHG-activity arises from the multipolar organic compound. This fact is in accordance with other reported second order optical measurements of similar octopolar systems [13]. In the same figure the SHG-response of the $\alpha$-quartz crystal (under similar experimental conditions, but in a fully phase-matched configuration) is presented. In fact, in case of the hybrid structure, a NLO-SHG conversion efficiency of about $27 \%$ of the reference sample is detected. This frequency doubling achieved by the hybrid sample represents in any case a remarkable SHGconversion efficiency. In order to give an estimate of the SHG conversion generated by the hybrid structure, a relative SHG-signal calibration was performed taking into account the SHG-signal provided by the reference crystal and using equation 1 . Due to the noncrystalline nature of the studied hybrid, the effective quadratic-NLO susceptibility $\chi_{\text {effs }}^{(2)}$ was evaluated to $13.6 \times 10^{-3} \mathrm{pm} / \mathrm{V}$ at $1064 \mathrm{~nm}$.

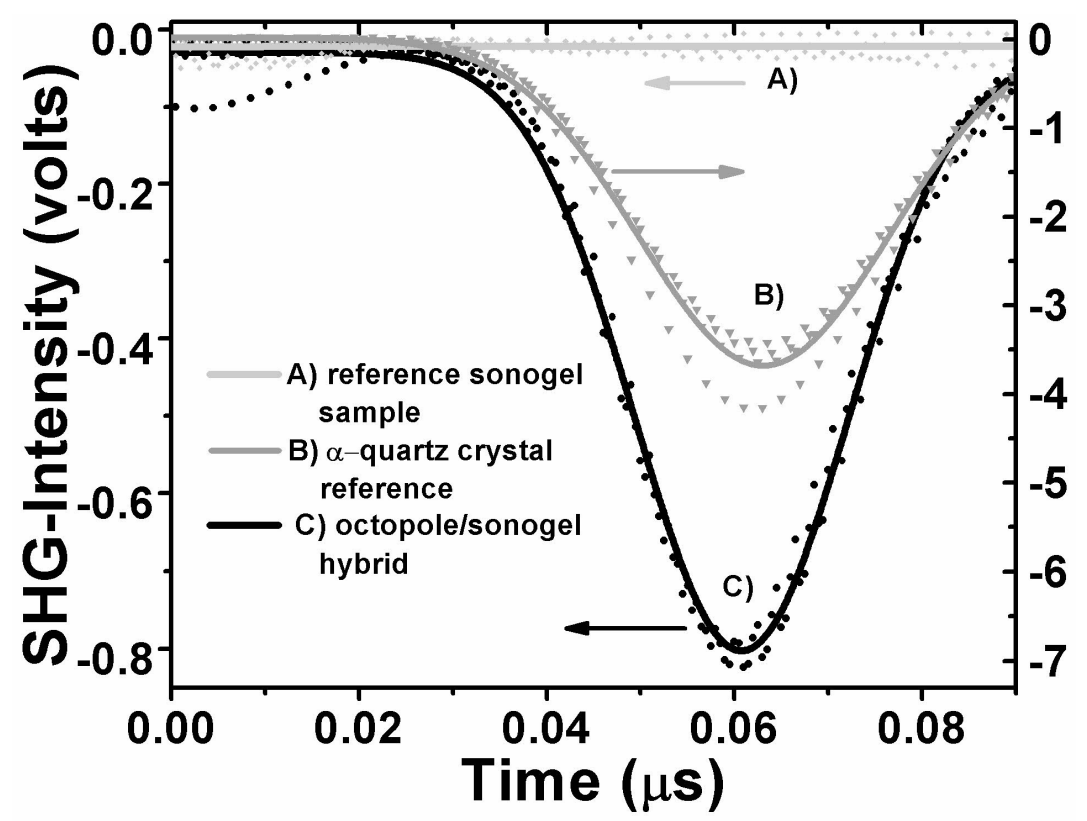

Figure 7. Comparative SHG transmission measurement in $\mathrm{P}_{\text {in }} / \mathrm{P}_{\text {out }}$ polarizing configuration, case of: A) pure reference sonogel, B) reference $\alpha$-quartz crystal, and C) octopole/sonogel composite. 
On the other hand, Figure 8 present the transition of SHG for different input polarization angles, the data distribution was linearly fitted with a correlation factor of 0.93 . This revels that the SHG-emissions of the amorphous organic-inorganic multipolar system do not depend on any polarization input, since the chromophores are randomly embedded within the glassy network and that the material inhomogeneities do not drastically affect the NLO-SHG-output signals. In fact, in this kind of composites, where multipolar molecular packing is present, a negligible dependence on polarizing and excitation geometries is observed.

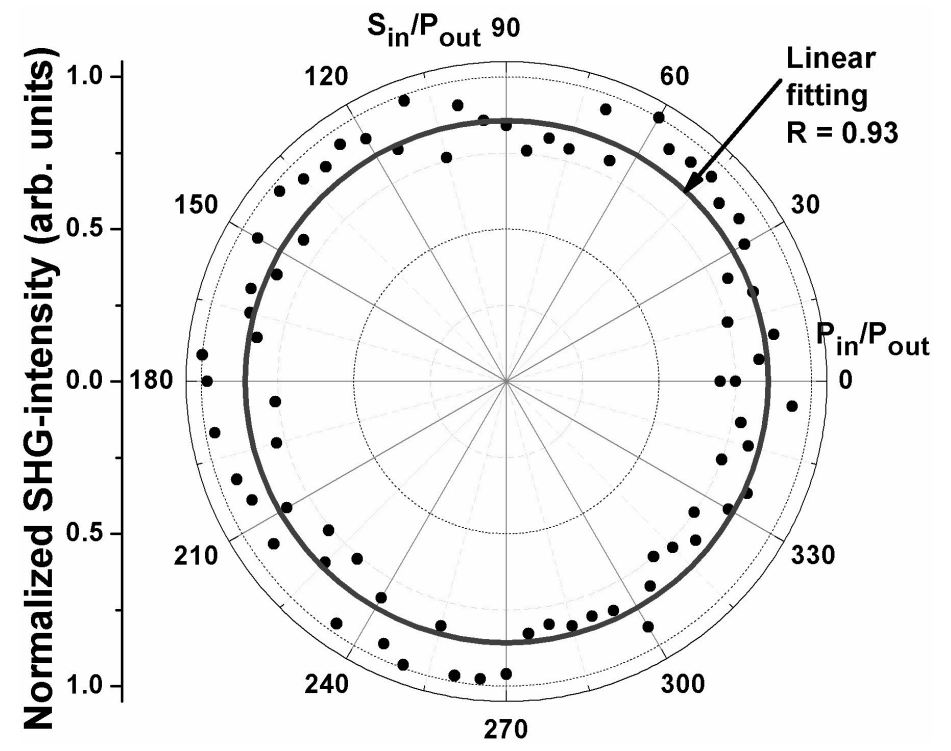

Figure 8. Polarizer vs. Analyzer angle dependent SHG-measurements for an octopole/sonogel hybrid glass.

\section{Conclusions}

The octopolar 1,3,5-tris(ethynylphenyl)benzene-(1) molecule was successfully embedded within the amorphous $\mathrm{SiO}_{2}$ sonogel network forming stable solid-state hybrid composites suitable for optical characterizations as demonstrated by several spectroscopic, linear and NLO-measurements and structural techniques. The large nanoporosity and surface areas obtained at room temperature from the sonogel glass, together with a stable mechanical performance achieved after drying, provide an excellent environment for the confinement of organic chromophores in rigid glassy composites in 
order to build robust guest-host systems pointing out to the development of new functionalized hybrid optical materials. Both, AFM and epimicroscopy techniques established an acceptable homogeneity in the surface and the bulk of the hybrid samples, respectively. UV-Vis- and PL-spectra have shown unambiguously evidence of homogeneous inclusion of the guest organic compound within the host $\mathrm{SiO}_{2}$ matrix. A red-shift in the main absorption band of the hybrid indicates the presence of $\mathrm{J}$ aggregates in the solid state; in addition, the presence of dopant octopolar compounds increases the PL-activity of the pure sonogel samples. On the other hand, without the necessity of chromophore alignment, the hybrid samples exhibit non-negligible quadratic NLO-activity on transmission, nonresonant SHG-experiments. In general, we can conclude that the octopolar chromophores embedded within the glassy network are dispersed to configure an isotropic and amorphous solid-state hybrid phase; however due to its inherent multipolar nature, these compounds are able to exhibit stable $\chi^{(2)}$ NLO-activity under any polarizing-excitation geometry. Further studies on the NLO properties

(including $\chi^{(3)}$-NLO properties) of several octopolar based hybrid systems should be performed in both bulk and thin film formats in order to provide a full optical and structural description of these materials and to explore potential photonic applications. These experiments are currently under way and will be presented in future works.

\section{Acknowledgments:}

We are grateful to J. Guadalupe Bañuelos (CCADET-UNAM) for valuable support on AFM-imaging and to Prof. G. Abbate for helpful discussions. O.G. Morales-Saavedra acknowledges financial support from the PAPIIT-DGAPA-UNAM (grant: IN-115508) and the SEP-CONACyT-MEXICO (grant: 82808-F). 


\section{References}

[1] Ledoux, I., \& Zyss, J. (2002). Comptes Rendus Physique, 3, 407.

[2] Cho, M.J., Choi, D.H., Sullivan, P.A., Akelaitis, A.J.P., \& Dalton, L.R. (2008). Progress in Polymer Science, 33, 1013.

[3] Asselberghs, I., Clays, K., Persoons, A., Ward, M.D., \& McCleverty, J. (2004). Journal of Materials Chemistry, 14, 2831.

[4] Cravino, A., Roquet, S., Alévêque, O., Leriche, P., Frère, P., \& Roncali, J., (2006). Chem. Mater., 18,2584 .

[5] De Bettignies, R., Nicolas, Y., Blanchard, P., Levillain, E., Nunzi, J.-M., \& Roncali, J., (2003). Adv. Mater., 15, 1939

[6] Asselberghs, I., Hennrich,G., \& Clays K., (2006). J. Phys. Chem., A, 110, 6271

[7] Feuvrie, C., Ledoux, I., Zyss, J., Le Bozec, H., \& Maury, O. (2005). Comptes Rendus Chimie, 8 (8 SPEC. ISS.), 1243

[8] Jeong, M.Y., Kim, H.M., Jeon, S. J., Brasselet, S., \& Cho, B.R. (2007). Advanced Materials, 19, 2107.

[9] Canva, M., Georges, P., Le Saux, G., Brun, A., Larrue, D., \& Zarzycki, J., (1991). J. Mat. Sci. Lett., 10, 615.

[10] Chaumel, F., Jiang, H., Kakkar, A. (2001). Chemistry of Materials, 13, 3389.

[11] Morales-Saavedra, O.G., Rivera, E., Flores-Flores, J.O., Castañeda, R., Bañuelos, J.G., \& Saniger, J.M., (2007). J. Sol-Gel Scie. Technol., 41(3), 277.

[12] Jia, C.Y., Que, W., \& Liu, W.G., (2009). Thin Solid Films, 518, 290.

[13] Hennrich, G., Omenat, A., Asselberghs, I., Foerier, S., Clays, K., Verbiest, T., \& Serrano, J.L., (2006). Angewandte Chemie - International Edition, 45, 4203. 
[14] Revillod, G., Russier-Antoine, I., Benichou, E., Jonin, C., \& Brevet, P.F. (2005). Journal of Physical Chemistry B, 109, 5383.

[15] , Scherer, G.W. (1990). "Sol-Gel Science: The Physics and Chemistry of Sol-Gel Processing", Academic Press, Boston.

[16] Sakka, Sumio, (Ed.), (2005). Handbook of Sol-Gel Science and Technology, (Processing, Characterization and Applications, V. I - Sol-Gel).

[17] Esquivias L, \& de la Rosa-Fox, N. (2003). J Sol-Gel Sci. Technol., 26, 651.

[18] Morales-Saavedra, O. G., Rivera, E. (2006). Polymer, 47(15), 5330.

[19] Kuzy, M. G., \& Dirk C. W. (Eds.), (1998). Characterization Techniques and Tabulations for Organic Nonlinear Optical Materials, Marcel Dekker, New York.

[20] Mortazavi, M.A., Knoesen, A., Kowel, S.T., Higgins, B.G. ,.\& Dienes, A,. J. (1989). Opt. Soc. Am. B, 6, 733 .

[21] Castañeda, L., Morales-Saavedra, O. G., Acosta, D. R., Maldonado, A., \& Olvera, M. (2006). Physica Status Solidi (a), 203(8), 1971.

[22] Kato, N., Yamamoto, K, \& Uesu, Y., (2007). Japanese Journal of Applied Physics, 46, 5318. 